Benchmarks

\section{A bacterial two-hybrid system that utilizes Gateway cloning for rapid screening of protein-protein interactions}

\author{
S.L. Rajasekhar Karna ${ }^{1}$, Xhavit Zogaj ${ }^{1}$, Jeffrey R. Barker ${ }^{1}$, Janakiram \\ Seshu ${ }^{1}$, Simon L. Dove ${ }^{2}$, and Karl E. Klose ${ }^{1}$ \\ ${ }^{1}$ South Texas Center for Emerging Infectious Diseases and Department of \\ Biology, University of Texas San Antonio, San Antonio, TX, USA and \\ ${ }^{2}$ Division of Infectious Diseases, Children's Hospital, Harvard Medical \\ School, Boston, MA, USA
}

BioTechniques 49:831-833 (November 2010) doi 10.2144/000113539

Keywords: Gateway cloning; bacterial two-hybrid; protein-protein interaction; Francisella; Vibrio

Supplementary material for this article is available at www.BioTechniques.com/article/113539.

Comprehensive clone sets representing the entire genome now exist for a large number of organisms. The Gateway entry clone sets are a particularly useful means to study gene function, given the ease of introduction into any Gateway-suitable destination vector. We have adapted a bacterial two-hybrid system for use with Gateway entry clone sets, such that potential interactions between proteins encoded within these clone sets can be determined by new destination vectors. We show that utilizing the Gateway clone sets for Francisella tularensis and Vibrio cholerae, known interactions between $F$. tularensis IglA and IglB and $V$. cholerae VipA and VipB could be confirmed with these destination vectors. Moreover, the introduction of unique tags into each vector allowed for visualization of the expressed hybrid proteins via Western immunoblot. This Gateway-suitable bacterial two-hybrid system provides a new tool for rapid screening of proteinprotein interactions.

Recently, complete sets of clones representing ORFs of a number of species have been constructed (1-3) utilizing Gateway entry clones and are available to investigators through the Pathogen Functional Genomics Resource Center (PFGRC; http://pfgrc.jcvi.org). Gateway cloning technology (Invitrogen, Carlsbad, CA, USA) uses $\lambda$ recombination proteins to circumvent traditional restriction enzyme/ligation cloning (4). ORFs are introduced into Gateway "entry vectors" and can then be moved into any appropriate Gateway-compatible "destination vectors" via $\lambda$-mediated recombination. The recombination reaction is rapid and allows for directional cloning that maintains reading frame.
We have developed new Gateway destination vectors for use in a bacterial two-hybrid system developed to detect interactions between fusions to a DNA binding protein and an RNA polymerase (RNAP)-binding protein (5-7). In the modified version of this system (8), interaction between a protein fused to Zif (Zinc-finger DNA binding domain from the murine $\mathrm{Zif} 268$ protein) and a protein fused to the $\omega$ subunit of Escherichia coli RNAP facilitates transcriptional activation of a Zif-dependent lac $Z$ reporter gene.

Type VI secretion systems (T6SSs) are recently described protein assemblies that facilitate secretion across Gram-negative bacterial membranes into the extracellular milieu and into eukaryotic cells (9-12).
The $V$. cholerae T6SS components VipA and $V$ ipB are essential for secretion and interact with each other to form a tubular complex $(13,14)$. Homologs of VipA and VipB are found in most T6SS gene clusters, including IglA and IglB of $F$. tularensis, which are encoded within the Francisella pathogenicity island and are essential for virulence. IglA and IglB have been shown to interact via immunoprecipitation (15) and a yeast two-hybrid system (14). The $\operatorname{IglAB} / \mathrm{Vip} A \mathrm{~B}$ interaction was therefore an ideal means to validate the new Gateway vectors designed to identify protein-protein interactions.

Incorporation of an ORF from a Gateway donor plasmid into pKEK1286 (pKEK1286 and pKEK1287 are described in the Supplementary Materials) results in a protein fusion at its $\mathrm{C}$ terminus with the zinc-finger DNA binding protein $\mathrm{Zif}$; it also incorporates a FLAG epitope at its $\mathrm{N}$ terminus (Figure 1). Incorporation of an ORF from a Gateway donor plasmid into pKEK1287 results in a protein fusion at its $\mathrm{C}$ terminus with the RNA polymerase $\omega$ subunit; it also incorporates a c-myc epitope at its $\mathrm{N}$ terminus.

We tested the utility of these bacterial two-hybrid destination vectors by examining protein-protein interactions between the $F$. tularensis IglA and IglB proteins. Entry clones containing IglA and IglB from the $F$. tularensis Gateway library at the PFGRC were introduced into pKEK1286 and pKEK1287, and the resulting protein fusions were analyzed for protein-protein interactions. Because the Gateway $\lambda$ recombination reactions require surrounding sequences that result in the introduction of additional amino acids between the ORF and the epitope tag and fusion partner (Zif or $\omega$ ) (Figure 1), we also introduced $i g l A$ and $i g l B$ into the original bacterial two-hybrid vectors by traditional cloning techniques (see Supplementary Materials) to determine if there were any negative effects of the linker regions in the Gateway-derived fusions. We then tested the cloned fusions $\left(\operatorname{IglA}^{\mathrm{C}}-\omega\right.$ and $\mathrm{IglB}^{\mathrm{C}}-\mathrm{Z}$ if $)$ along with the Gateway-derived IglA and IglB fusions ( $\operatorname{IglA}^{\mathrm{G}}-\mathrm{Zif}, \operatorname{IglA}^{\mathrm{G}}-\omega, \mathrm{IglB}^{\mathrm{G}}-\mathrm{Z}$ if, and $\left.\operatorname{IglB}^{\mathrm{G}}-\omega\right)$ for their ability to interact (Figure 2).

The IglA-Zif and IglB- $\omega$ protein fusions created by restriction/ligation $\left(\mathrm{IglA}^{\mathrm{C}}-\mathrm{Zif}\right.$, $\left.\mathrm{IglB}^{\mathrm{C}}-\omega\right)$ interact and stimulate transcription from the engineered promoter-lac $Z$ fusion present in the KDZif1 $\Delta Z$ E. coli reporter strain, as anticipated. Likewise, the IglA-Zif $+\operatorname{IglB}-\omega$ and IglB-Zif $+\operatorname{IglA}-\omega$ protein fusions created in the new Gateway destination vectors $\left(\operatorname{Igl} A^{\mathrm{G}}-\mathrm{Zif}+\operatorname{IglB}^{\mathrm{G}}-\omega\right.$ and $\operatorname{IglA}^{G}-\omega+\operatorname{IglB}^{G}-$ Zif) also interact and 

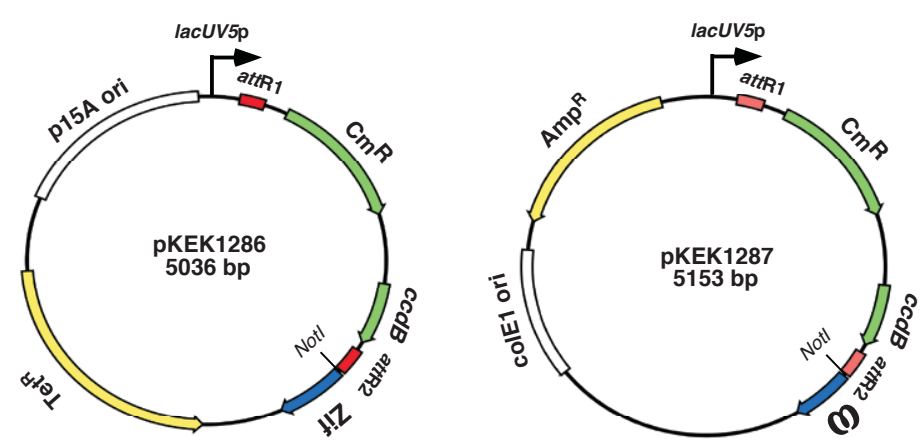

pKEK1286

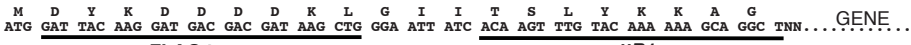
FLAG tag attB

GENE $\begin{array}{llllllllllllllllllllll}\text { P } & \text { A } & \text { F } & \text { L } & \text { X } & \text { K } & \text { V } & \text { V } & \text { I } & \text { K } & \text { L } & \text { E } & \text { A } & \text { A } & \text { A } & \text { P } & \text { R } & \text { V } & \text { R } & \text { T } & \text { G } & \text { S }\end{array}$ attB2

Notl

Zif

pKEK1287

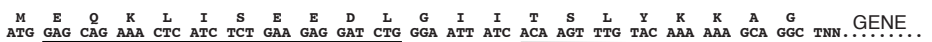
c-myc tag attBi

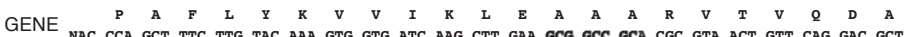
attB2

Noti

$\omega$

Figure 1. Bacterial two-hybrid Gateway destination vectors. Shown are the attributes of pKEK1286 and pKEK1287 (top) and sequences of the expected products following the Gateway recombination reaction into these plasmids (bottom).

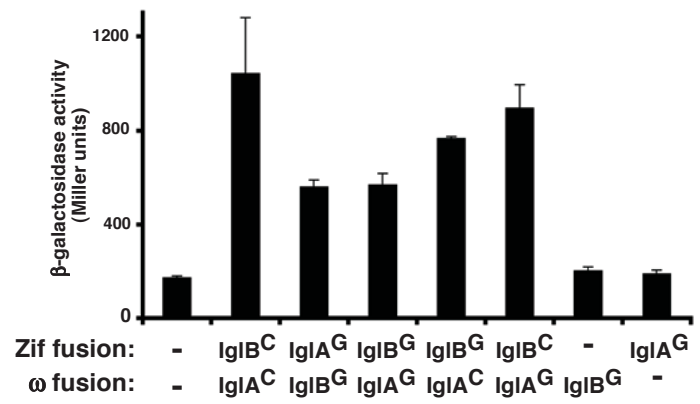

Figure 2. Gateway bacterial two-hybrid analysis of $\boldsymbol{F}$. tularensis IgIA and IgIB interaction. E. coli reporter strain KDZif1 $\triangle Z$ was transformed with empty

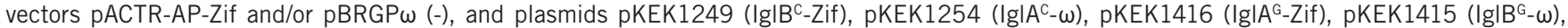
pKEK1417 (Ig|A $\left.A_{-}-\omega\right)$, and/or pKEK1414 (Ig|B $\left.{ }^{G}-Z i f\right)$, and assayed for $\beta$-galactosidase activity. C superscript denotes cloned protein fusions, while $G$ superscript denotes protein fusions created by Gateway LR reaction. All values result from triplicate samples, and IgIA + IgIB interactions gave values significantly above background (either empty vectors or single plasmid paired with empty vectors; $P<0.001$, Student's $t$-test).

stimulate transcription in the reporter strain; the interaction is independent of whether IglA or IglB is fused to Zif or $\omega$. Either IgA-Zif or IglB- $\omega$ fusions paired with empty vectors resulted in background levels of activity.

The Gateway-derived fusions exhibited reduced activity when compared with the cloned fusions. Pairing a Gateway-derived protein fusion with its cognate cloned protein fusion $\left(\operatorname{IglA}^{\mathrm{C}}-\mathrm{Z}\right.$ if $+\operatorname{IglB}^{\mathrm{G}}-\omega$ and $\mathrm{IglA}^{\mathrm{G}}-\mathrm{Zif}+\mathrm{IglB}^{\mathrm{C}}-\omega$; Figure 2) led to an intermediate level of activity. We can conclude that this lower activity is linked to the Gateway-derived protein fusions and is due to the presence of the additional amino acids in these fusions introduced in the linker regions required for $\lambda$ recombination and/or the epitope tags on the $\mathrm{N}$ termini (Figure 1). Still, the interaction between the Gateway-derived protein fusions is significantly elevated above background and therefore provides a useful new tool to study protein-protein interactions.

We further measured protein-protein interactions between the $V$. cholerae VipA and $\mathrm{VipB}$ proteins, which are homologs of the F. tularensis IglA and IglB proteins (13). Entry clones containing VipA and VipB from the $V$. cholerae Gateway library were introduced into pKEK1286 and pKEK1287, and the resulting protein fusions were analyzed for protein-protein interactions (Supplementary Figure S1A). The Gateway-derived IglA- $\omega$ and IglB-Zif fusions were utilized as interacting controls. The VipA- $\omega$ and VipB-Zif fusion proteins interact and stimulate significant $\beta$-galactosidase activity. No cross-species 
interactions could be detected between IglA- $\omega$ and VipB-Zif or between IglB-Zif and VipA- $\omega$; the level of $\beta$-galactosidase activity in the reporter strain containing these fusions was not significantly different than the activity in strains containing just VipA- $\omega$ or VipB-Zif. This confirms previous results, where no interactions could be detected between IglA and VipB or IglB and VipA (14).

The $\mathrm{N}$-terminal epitope tags in the Gateway-derived protein fusions were introduced to facilitate detection via Western immunoblot. To determine the utility of these tags, whole cell lysates of mid-log cultures used to measure VipA-VipB interactions were subjected to Western immunoblot analysis with anti-FLAG and anti-cmyc monoclonal antibodies (Supplementary Figure S1B). Specific detection of the fusion proteins was observed with antic-myc $(\sim 33 \mathrm{kDa})$ and anti-FLAG $(\sim 72 \mathrm{kDa})$ antibodies, as anticipated.

The new bacterial two-hybrid Gateway destination vectors described here should prove useful in the screening of complete genome Gateway clone libraries for proteinprotein interactions. The ease of incorporation of Gateway entry clone ORFs into destination vectors should allow highthroughput techniques to be utilized in combination with these vectors.

\section{Acknowledgments}

This study was supported by the National Institutes of Health (NIH; grant nos. P01 AI57986 and R01 AI43486, to K.E.K.). This paper is subject to the NIH Public Access Policy.

\section{Competing interests}

The authors declare no competing interests.

\section{References}

1.Matsuyama, A. and M. Yoshida. 2009. Systematic cloning of an ORFeome using the Gateway system. Methods Mol. Biol. 577:11-24.

2.Aguiar, J.C., J. LaBaer, P.L. Blair, V.Y. Shamailova, M. Koundinya, J.A. Russell, F. Huang, W. Mar, et al. 2004. Hig-throughput generation of $P$. falciparum functional molecules by recombinational cloning. Genome Res. 14:2076-2082.

3. Gao, H., D. Pattison, T. Yan, D.M. Klingeman, X. Wang, J. Petrosino, L. Hemphill, X. Wan, et al. 2008. Generation and validation of a Shewanella oneidensis MR-1 clone set for protein expression and phage display. PLoS One 3:e2983.

4.Walhout, A.J., G.F. Temple, M.A. Brasch, J.L. Hartley, M.A. Lorson, S. van den
Heuvel, and M. Vidal. 2000. Gateway recombinational cloning: application to the cloning of large numbers of open reading frames or ORFeomes. Methods Enzymol. 328:575-592.

5.Dove, S.L. and A. Hochschild. 1998. Conversion of the omega subunit of Escherichia coli RNA polymerase into a transcriptional activator or an activation target. Genes Dev. 12:745-754.

6. Dove, S.L., J.K. Joung, and A. Hochschild. 1997. Activation of prokaryotic transcription through arbitrary protein-protein contacts. Nature 386:627-630.

7.Joung, J.K., E.I. Ramm, and C.O. Pabo. 2000. A bacterial two-hybrid selection system for studying protein-DNA and protein-protein interactions. Proc. Natl. Acad. Sci. USA 97:7382-7387.

8. Vallet-Gely, I., K.E. Donovan, R. Fang, J.K. Joung, and S.L. Dove. 2005. Repression of phase-variable cup gene expression by H-NS-like proteins in Pseudomonas aeruginosa. Proc. Natl. Acad. Sci. USA 102:11082-11087.

9. Pukatzki, S., A.T. Ma, D. Sturtevant, B. Krastins, D. Sarracino, W.C. Nelson, J.F. Heidelberg, and J.J. Mekalanos. 2006. Identification of a conserved bacterial protein secretion system in Vibrio cholerae using the Dictyostelium host model system. Proc. Natl. Acad. Sci. USA 103:1528-1533.

10.Mougous, J.D., M.E. Cuff, S. Raunser, A. Shen, M. Zhou, C.A. Gifford, A.L. Goodman, G. Joachimiak, et al. 2006. A virulence locus of Pseudomonas aeruginosa encodes a protein secretion apparatus. Science 312:1526-1530.

11.Ma, A.T., S. McAuley, S. Pukatzki, and J.J. Mekalanos. 2009. Translocation of a Vibrio cholerae type VI secretion effector requires bacterial endocytosis by host cells. Cell Host Microbe 5:234-243.

12.Barker, J.R., A. Chong, T.D. Wehrly, J.-J. Yu, S.A. Rodriguez, J. Liu, J. Celli, B.P. Arulanandam, and K.E. Klose. 2009. The Francisella tularensis pathogenicity island encodes a secretion system that is required for phagosome escape and virulence. Mol. Microbiol. 74:1459-1470.

13. Bonemann, G., A. Pietrosiuk, A. Diemand, H. Zentgraf, and A. Mogk. 2009. Remodelling of VipA/VipB tubules by ClpVmediated threading is crucial for type VI protein secretion. EMBO J. 28:315-325.

14. Broms, J.E., M. Lavander, and A. Sjostedt. 2009. A conserved alpha-helix essential for a type VI secretion-like system of Francisella tularensis. J. Bacteriol. 191:24312446.

15.de Bruin, O.M., J.S. Ludu, and F.E. Nano. 2007. The Francisella pathogenicity island protein IglA localizes to the bacterial cytoplasm and is needed for intracellular growth. BMC Microbiol. 7:1.

Received 19 July 2010; accepted 27 September 2010.

Address correspondence to Karl E. Klose, Department of Biology, University of Texas San Antonio, 1 UTSA Circle, San Antonio, TX, 78249, USA.e-mail: karl.klose@utsa.edu

To purchase reprints of this article, contact: carmelitag@fosterprinting.com

\section{BioTechnioues}

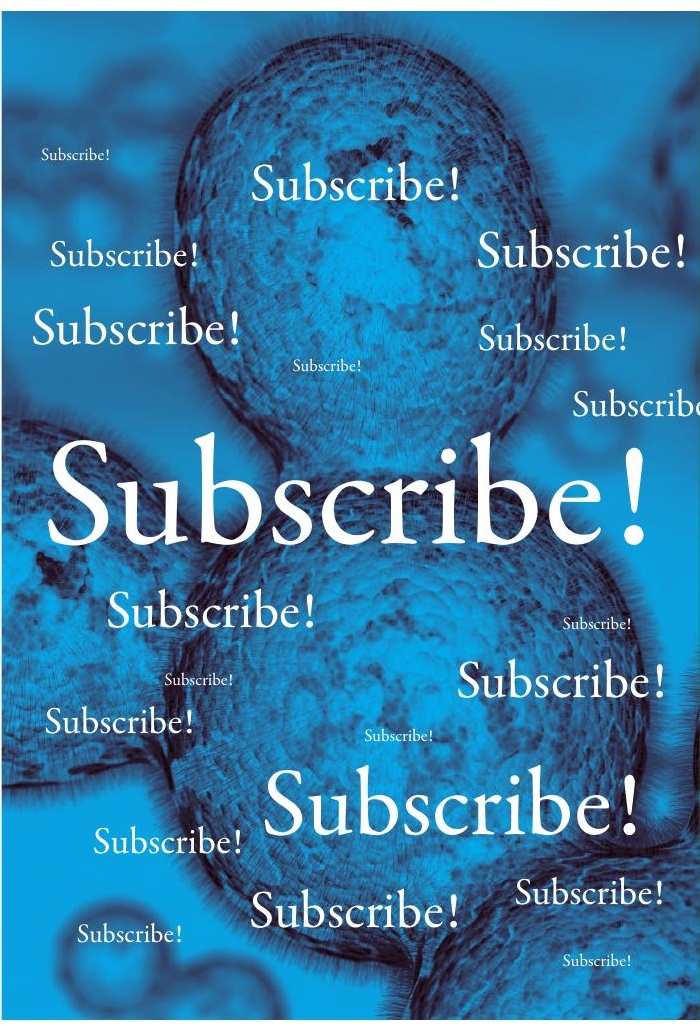

BioTechniques is the first peer-reviewed journal with open access to the whole of the life science community.

$100 \%$ life science

$100 \%$ peer-reviewed methods $100 \%$ "everyday" practical information

Maybe it's time you get your own free subscription copy too, or maybe your subscription has recently expired. Don't worry, it's easy and free to subscribe or renew to BioTechniques.

Go to:

www.BioTechniques.com/subscribe 